\title{
Histologic examination of the clipped internal thoracic artery by transmission electron microscope in patients undergoing coronary artery bypass grafting
}

\author{
Cenap Ozkara
}

Department of the Cardiovascular Surgery, Corlu Sifa Hospital, Tekirdag, Turkey

Email: cenapozkara@corlusifa.com

Received 30 March 2012; revised 3 May 2012; accepted 6 June 2012

\begin{abstract}
Objectives: As we known that the internal thoracic artery (ITA) has an excellent patency rate in coronary artery bypass grafting (CABG). However, early graft failure due to occlusion and spasm is still the major problem after coronary artery bypass surgery. We examined histopathologic findings of the clipped internal thoracic artery (ITA) in patients undergoing CABG using transmission electron microscope (TEM). Methods: To investigate the histopathololojic ITA examination, 60 patients were randomly selected. The ITA was harvested in a standart fashion with the use of low voltage electrocotery and its distal end was cut prior to bifurcation and clipped. Just before the ITA anastomosis $1 \mathrm{~mm}$ lenght of ITA ring was cut and saved in $2.5 \%$ Glutaraldehide solution for fixation and examination of transmission electron microscope (TEM). One blinded anatomopatholog examined all specimens and described the endothelial integrity according to the score system proposed by Fischlein et al. Results: In ten cases (17.5\%) different degree of histopathologic findings (endothelial cells, intercellular and intracellular organels and adventitia) were recorded The most important histopatholojic findings of ITA were as follows: endothelial vacuolisation, intimal thickening and/or intimal seperation, subendothelial edema, swallowing of cytoplasma and mitocondria. Conclusion: Our results showed that the endothelial pathology is high when the ITA clipped. Unfortunataly, because our study did not include the non-clipped ITA we have not concluded the comparison or statistical results. In our opinion, for absolute definition of the effects of the clipping the ITA it should be planned the comparative results between the clipped and non-clipped ITA histologic examinations.
\end{abstract}

Keywords: Internal Thoracic Artery; ITA Clipping;
Endothelia Pathology

\section{INTRODUCTION}

To date, the internal thoracic artery has been the most reliable conduit in coronary artery bypass surgery. However, graft thrombosis and spasm can be seen after coronary bypass operations. Endothelial damage during the harvesting is one the cause of these complications. When an injury develop coagulation cascade is activated. Thus, early graft thrombosis or spasm can be seen [1]. The damage to the endothelium can also promote the accelerated atherosclerotic process [2], thus eventually leading to the long-term development of graft stenosis or occlusion. In traditional technique, the surgeons prepares the ITA in a pedicle, cutting it at the distal end, occluding it with a clip and storing it in a vasodialtor-soaked tissue until it is implanted.

The aim of our study is to evaluate the pathology of the endothelial integrity of the ITAs in our CABG patients.

\section{MATERIAL AND METHODS}

Preoperatively, informed consent was obtained from all patients. The left ITA harvested from 60 consecutive patients undergoing elective CABG were the subject of this study. The mean age of the patient was $66 \pm 3.2$ year (min.: 48; max.: 94y). 75\% of cases were male. Smoking history was noted in $64 \%$ of patients. The most of the patients had multivessel coronary heart disease. 8 patients had only left anterior descending coronary artery stenosis. 57\% of cases had hypertension and $66 \%$ of cases had diabetes mellitus. 53\% of patients have been administered oral antidiabetics and the remaining cases have been medicated with insuline. All patients have been treated using antiaggregant therapy (asethyl salisilic ascite), beta blocker agents and nitrates preoperatively. Patients' charecteristics are summarised in Table 1. In all patients, the ITA was harvested with the pedicle using a 
Table 1. Composition of study group including general data and medical treatment.

\begin{tabular}{lc}
\hline Number of patients & 60 \\
\hline Age (years) & $162(148$ - 182) \\
Height $(\mathrm{cm})$ & $68(58-117)$ \\
Weight $(\mathrm{kg})$ & $22(19-33)$ \\
BMI $\left(\mathrm{kg} / \mathrm{m}^{2}\right)$ & Preparation to implantation mean: 49 \\
Time & (min.: 29, max.: 58) \\
Smoker & $64 \%$ \\
Hypertension & $57 \%$ \\
Diabetes & $66 \%$ \\
Hypercholesterolemia & $46 \%$ \\
Beta-Blocker Therapy & $100 \%$ \\
Calcium Antagonists & $80 \%$ \\
ACE Inhibitors & $55 \%$ \\
Aggregation Inhibitors & $100 \%$ \\
Anticoagulants & $25 \%$ \\
Coronary Vasodilators & $100 \%$ \\
Diuretics & $20 \%$ \\
\hline
\end{tabular}

low voltage electrocotery.

\subsection{Operative Technique}

ITA conduit was harvested by one surgeon. After performing a standard midline sternotomy, the left ITA was harvested in a pedicled fashion. A longitudinal incision on the endothoracic fascia about $1 \mathrm{~cm}$ medial to the ITA and its medial satellite vein using electrocoter was made. The branches of the ITA were clearly visualized. A least 2 - $3 \mathrm{~mm}$ away from the ITA itself the branches were clipped, we were thus able to divide the branch. During conduit harvesting, care should be taken that the elecrocotery does not come in contact with the ITA. In this way, the ITA is fully harvested from its origin to $1 \mathrm{~cm}$ beyond the bifurcation. After the administration of full dose heparin, the terminal portion of the ITA just proximal to the bifurcation was cut. Occluding it with a clip and storing it in a vasodialtor-soaked tissue until it is implanted. $1 \mathrm{~mm}$ ITAs' ring has been provided just before the anastomosis to the LAD artery to evaluate its endothelial integrity and electron microscobic examination.

\subsection{Preparation for Tranmission Electron Microscopy}

The ITA cylinders were cut longitudinally just before the anastomosis and then were immediately washed gently with a physiologic solution, and immersed in $2.5 \%$ glutaraldehyde. All samples were fixed in $2.5 \%$ glutaraldehyde for $24 \mathrm{~h}$ washed in phosphate buffer (PH: 7.4), postfixed in $1 \%$ osmium tetroxide $\left(\mathrm{OsO}_{4}\right)$ in phosphate buffer, and thereafter were further dehydrated in ascending concentrations of ethyl alcohol. Then, the tissues were washed with propylene oxyde and embedded in epoxy-resin embedding media. Semi-thin sections about $2 \mu \mathrm{m}$ in thickness and ultrathin sections about $60 \mathrm{~nm}$ in thickness were cut with a glass knife on a LKB-Nova (Sweden) ultramicrotome. Semi-thin sections were satined with methylene blue and examined by a Nikon Optiphot (Japan) light microscope. Ultrathin sections were collected on copper grids, stained with uranyl acetate and lead citrate and examined with a Jeol JEM 1200EX (Japan) transmission electron microscope and finally were observed. One blinded anatomopatholog examined all specimens and described the endothelial integrity according to the score system proposed by Fischlein et al. [3] using the following criteria: 1) completely confluent endothelium; 2) partially confluent endothelium; 3) loosely netted endothelium; 4) islands of endothelium; and 5) no endothelium.

\section{RESULTS}

In ten cases, there were different degrees of pathologic conditions in ITA examination. The main pathologic findings in these tissues were as follows.

A significant decrease in smooth muscle celss was observed. Swollen mitochondria were present. There was a heterochromatine rich nucleus in these cells. The cytoplasm of these cells swollen due to the collection of fluid in 6 patients (Figure 1). The normal endothelial morphology was seen in 50 patients. Normal endothelial configuration is seen in Figure 2. There is no any evidence for endothelial or subendothelial pathology in these 50 patients. Subendothelial vacuolisation and endothelial cell seperation was seen in 5 patients. Edematous areas were present around the external elastic membrane in tunica media and tunica adventitita in these 5 patients (Figure 3). An edema was present around the internal elastic membrane in 3 patients. This was subendothelial and pericellular edema of smooth muscle cells of tunica media. Additionally, swollen mitocondria were seen in the cytoplasm of smooth muscle cells in 4 patients. The cellular damage and seperation due to severe edema was present in between the smooth muscle cells of tunica media in 3 patients. Subendothelial swallowing and cytosceletal damage as a main pathologic finding was recorded in 2 patients. The severely seperation of the endothelial layers was detected in one patient. This case was 68 years old male and was diabetic. It has been showed that the adventitia seperated from the subendothelial layer (Figure 4).

\section{DISCUSSION}

It is now well established that using the internal thoracic 


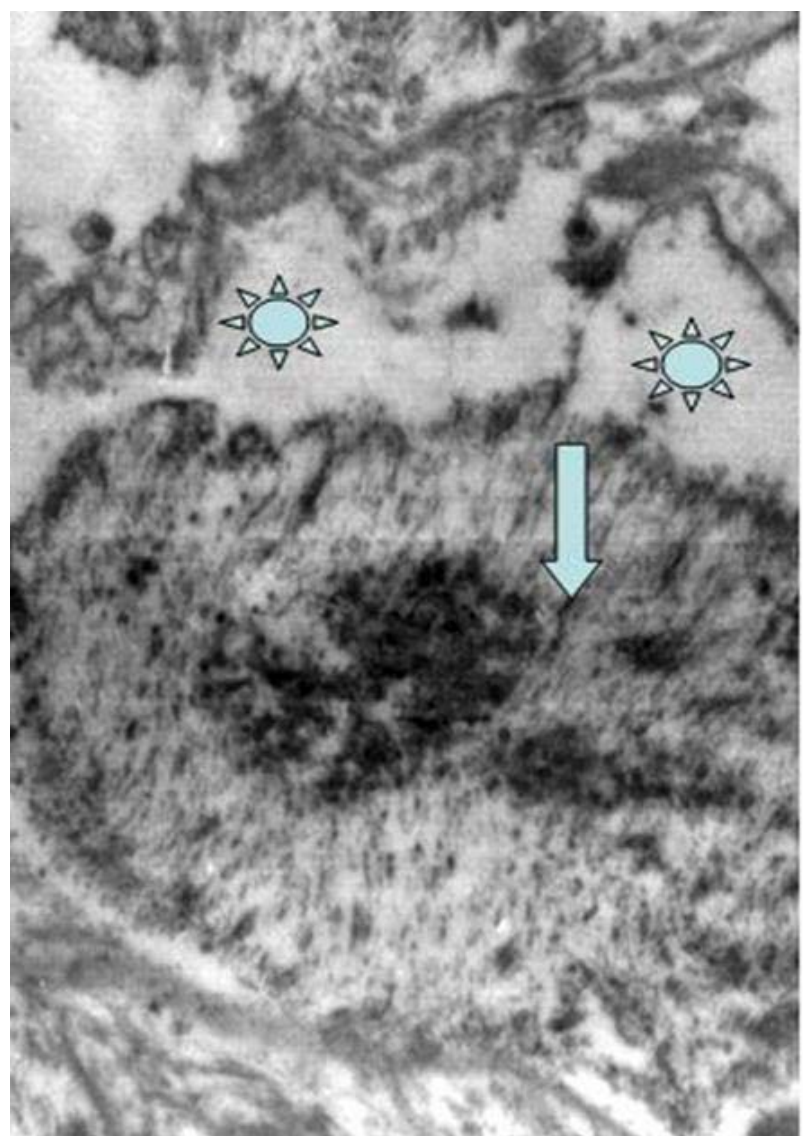

Figure 1. A significant decrease in smooth muscle celss of tunica media is observing in the intracellular organelles. A heterochromatine rich nucleus in these cells is also note (arrow head). The cytoplasm of these cells swollen due to the collection of fluid. Subendothelial vacuolisation and endothelial cell seperation is exhibiting in this figure (markers). Swollen mitochondria was also seen.

artery has a long-lasting impact on survival after CABG [3-5]. Thrombosis and/or vasospasm of the ITA in the postoperative period $[6,7]$ and the development of intimal proliferation and atherosclerosis years after coronary artery bypass surgery have previously been reported [6]. Both of these problems may lead to adverse outcomes for the patient. In the previous investigation, it has been shown that cellular loss and damages of intercellular region could be seen in the clipped ITA [8]. Grapow et al. have found that the concentrations of sP-selectin and thrombomodulin were significantly higher in plasma from clipped arteries compared to perfused arteries [8]. Scanning electron microscopic examination have revealed significant structural changes and loss of endothelial cells in the clipped arteries in their 3 patients. In contrast to perfused ITA, clipped arteries have showed severe endothelial cell damage and cell loss with exposure of basal lamina in this investigation. Depositions of cellular remnants and fibres were more frequently seen in the clipped artery, additionally.

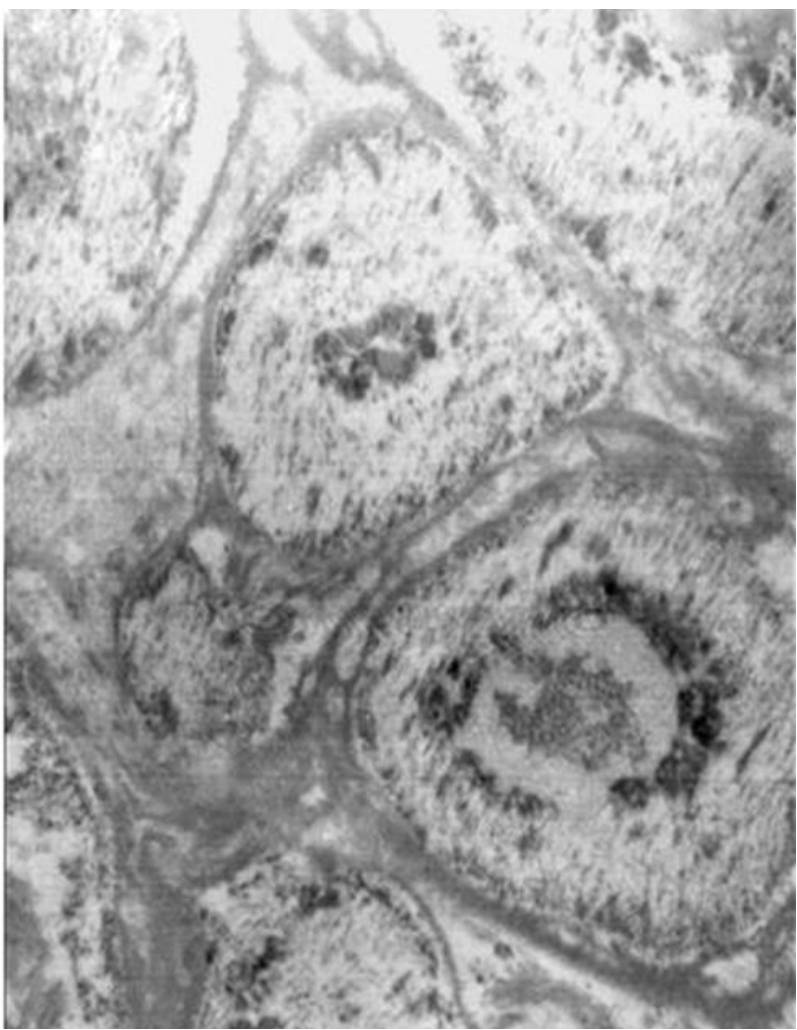

Figure 2. Demonstrating the normal ITA hystologic examination and cells configuration.

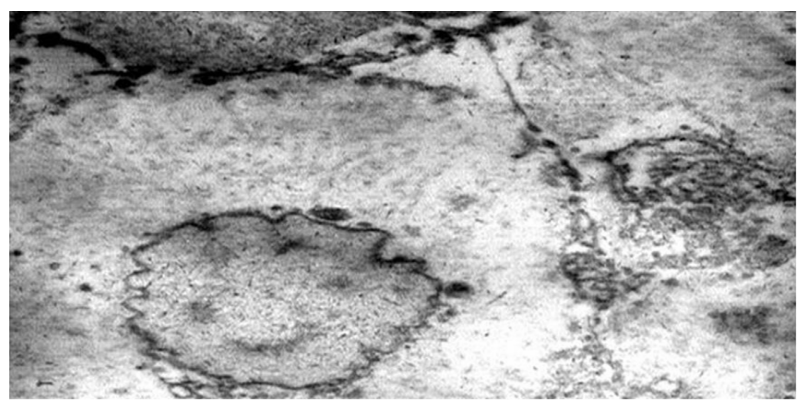

Figure 3. Shows edematous cytoplasma and heterochromatine rich nucleus.

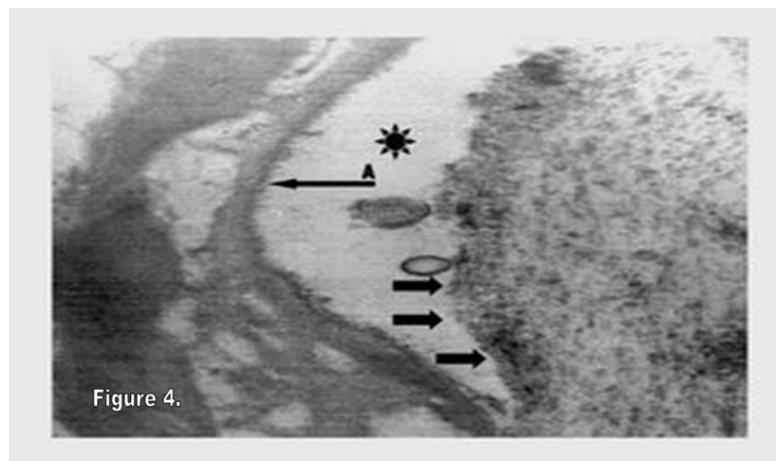

Figure 4. Exhibiting the severely seperation of the endothelial layers. Arrow A is demonstrating the adventitia which seperated from the subendothelial layer. The black sunny signe. 
It is known that the arterial endothel plays an important role in vascular tone since it releases autocrine and paracrine substances such as internal vasodilators and prostacycline. To mediating vasodilation, the endothelium exerts antiatherogenic effects through potent inhibition of platelet aggregation, smooth-muscle proliferation, and leukocyte adhesion $[9,10]$.

In traditional technique the surgeons prepares the bypass conduit in a pedicle, cutting it at the distal end, occluding it with a clip and storing it in a vasodialtorsoaked tissue until it is implanted. Especially in a multivessel coronary bypass operations we need long time from harvesting to implantation of the graft. Thus, some mediators such as selectin family members are excreated and nitric oxyde level is decreased due to luminal high pressure. In our investigation, the time period between the harvesting and implantation of the ITA conduit was mean: $49 \mathrm{~min}$. The time period from harvesting to anastomosis was shortly in cases who had one coronary artery disease (in 5 cases). However, in 3 of 5 patients with one coronary artery disease ITA pathology has also been recorded. This result has been shown that endothelial damage is not depend on the waiting period.

The other problem is the usage of harvesting devices during the ITA preperation. The histological study confirmed that a different devices such as ultrasonic scalpel or high frequency electrocotery application on the branches more than 1 or $5 \mathrm{~mm}$ away from the ITA itself did not cause any endothelial injury [11,12]. Therefore, our investigation was done by carrefully observation during the harvesting with the aid of low voltage electrocotery. Grapow et collegues compared the two groups of harvested ITA using different harvesting techniques includeing clipped and perfused ITA storage. In this study, the authors have showed that leaving the ITA perfused during harvesting could improve considerably the endothelial function of the graft. They have reported that the clipped ITA had higher contractile responses compared to perfused ITA. But, they did not report the endothelial integrity and ultrastructurall changes of the conduits [13].

\section{CONCLUSIONS}

In conclusion, the ITA graft is generally regarded as the standard conduit for CABG because of its excellent patency and low prevalence of histopathologic changes. The vascular endothelium is of primary importance in maintaining viability and patency of ITA. Surgical manipulation, ischemia, storage conditions, and distension before anastomosis can abnormally alter the antithrombogenic property of the endothelium leading to vasospasms, thrombogenesis, occlusive intimal hyperplasia, and stenosis. Intimal hyperplasia occurs as a consequence of physiological stimuli, constituting an attempt by the tissue to maintain normal conditions of flow, wall tension or both. In our study, ultrastructural ITA pathology including subadventitial region, cellular and intercellular relation is high. This may be due to high intraluminal conduit pressure. However, because we have not researched the perfused ITA our study results is not comparative. Thus, for definitive conclusion we need a comparative structural and morphologic result between the clipped and perfused ITA conduits. Our research is continued for the perfused ITA in our CASBG patients for comparison of the clipped ITA.

On the other side, our study includes largest cases for examining the ultrastructural intracellular and cellular organel pathology of the clipped ITA conduits' walls using a TEM in the English literature. In the present study, we morphologically and ultrastructurally assessed the endothelial integrity of the ITA using TEM in our 60 CABG cases. According to our results and previously published reports we don't suggest the ITA clipping in CABG operations.

\section{REFERENCES}

[1] Thatte, H.S. and Khuri, S.F. (2001) The coronary artery bypass conduit: I. Intraoperative endothelial injury and its implication on graft patency. Annals of Thoracic Surgery, 72, S2245-S2252. doi:10.1016/S0003-4975(01)03272-6

[2] Verrier, E.D. and Boyle, E.M. Jr. (1996) Endothelial cell injury in cardiovascular surgery. Annals of Thoracic Surgery, 62, 915-922. doi:10.1016/S0003-4975(96)00528-0

[3] Fischlein, T., Schütz, A., Uhlig, A., Frey, R., Krupa, W., Babic, R., Thiery, J. and Reichart, B. (1994) Integrity and viability of homograft valves. European Journal CardioThoracic Surgery, 8, 425-430. doi:10.1016/1010-7940(94)90084-1

[4] Loop, F.D., Lytle, B.W., Cosgrove, D.M., et al. (1986) Influence of the internal-mammary artery graft on 10year survival and other cardiac events. The New England Journal of Medicine, 314, 1-6. doi:10.1056/NEJM198601023140101

[5] Grapow, M.T.R., Preiss, M., Bernet, F. and Zerkowski, H.R. (2002) Surgical treatment in end-stage coronary artery disease. Cardiovascular Medicine, 5, 190-197.

[6] Sarabu, M.R., McClung, J.A., Fass, A. and Reed, G.E. (1987) Early postoperative spasmin left internal mammary artery bypass grafts. Annals of Thoracic Surgery, 44, 199-200. doi:10.1016/S0003-4975(10)62041-3

[7] Shelton, M.E., Forman, M.B., Virmani, R., Bajaj, A., Stoney, W.S. and Atkinson, J.B. (1988) A comparison of morphologic and angiographic findings in long-term internal mammary artery and saphenous vein bypass grafts. Journal of the American College of Cardiology, 11, 297-307. doi:10.1055/s-2005-865827

[8] Grapow, M.T.R., Konerding, M.A., Müller-Schweinitzer, E., Bernet, F., Matt, P., Reineke, D.C. and Zerkowski, H.R. (2005) Protecting the endothelial integrity of internal 
thoracic arteries. The Thoracic and Cardiovascular Surgeon, 53, 352-357.

[9] Luscher, T.F. and Noll, G. (1995) The pathogenesis of cardiovascular disease: Role of the endothelium as a target and mediator. Atherosclerosis, 118, 81-90. doi:10.1016/0021-9150(95)90076-4

[10] Moncada, S. and Higgs, A. (1993) The L-arginine-nitric oxide pathway. The New England Journal of Medicine, 329, 2002-2012. doi:10.1056/NEJM199312303292706

[11] Stary, H.C. (1992) Composition and classification of human atherosclerotic lesions. Virchows Archiv A, Pathological Anatomy and Histopathology, 421, 277-290. doi:10.1007/BF01660974
[12] Higami, T., Maruo, A., Yamashita, T., Shida, T. and Ogawa, K. (2000) Histologic and physiologic evaluation of skeletonized internal thoracic artery harvesting with an ultrasonic scalpel. Journal of Thoracic and Cardiovascular Surgery, 120, 1142-1147. doi:10.1067/mtc.2000.110189

[13] Grapow, M.T., Kern, T., Reineke, D.C., Brett, W., Bernet, F., Rueter, F., Muller-Schweinitzer, E. and Zerkowski, H.R. (2003) Improved endothelial function after a modified harvesting technique of the internal thoracic artery. European Journal Cardio-Thoracic Surgery, 23, 956-960. doi:10.1016/S1010-7940(03)00122-2 